\title{
Effect of non-sperm cells removal with single-layer colloidal centrifugation on myeloperoxidase concentration in post-thaw equine
} semen

\author{
Jérôme Ponthier ${ }^{a, *}$, Sheila R. Teague ${ }^{c}$, Thierry Y. Franck ${ }^{b}$, Geoffroy de la Rebière ${ }^{a, b}$, \\ Didier D. Serteyn ${ }^{\mathrm{a}, \mathrm{b}}$, Steven P. Brinsko ${ }^{\mathrm{c}}$, Charles C. Love ${ }^{\mathrm{c}}$, Terry L. Blanchard ${ }^{\mathrm{c}}$, \\ Dickson D. Varner ${ }^{\mathrm{c}}$, Stéfan C. Deleuze ${ }^{\mathrm{a}}$ \\ ${ }^{a}$ Equine Clinic, Veterinary Medicine Faculty, ULg, University of Liège, Liège, Belgium \\ ${ }^{\mathrm{b}}$ Center for Oxygen Research and Development (CORD), ULg University of Liège, Liège, Belgium \\ ${ }^{\mathrm{c}}$ Departement of Large Animal Clinical Sciences, College of Veterinary Medicine and Biomedical Sciences, Texas AE'M University, College Station, Texas, USA
}

\section{A R T I C L E I N F O}

\section{Article history:}

Received 18 April 2013

Received in revised form 6 August 2013

Accepted 10 August 2013

\section{Keywords:}

Stallion

Semen

Cryopreservation

Non-sperm cells

Myeloperoxidase

Colloid centrifugation

\begin{abstract}
A B S T R A C T
Myeloperoxidase (MPO) is a pro-oxidant enzyme contained in and released by neutrophils during degranulation or after lysis. Post-thaw semen contains MPO and its concentration is associated with decreased sperm motility. Recently, MPO concentration in post-thaw semen was shown to be associated with the presence of non-sperm cells (NSC). The objective of this study was to evaluate the effect of a single-layer colloidal centrifugation before cryopreservation on NSC and MPO concentrations in equine semen. The experimental design consisted of freezing semen with or without previous centrifugation through two concentrations of single-layer colloid media. Non-sperm cells and MPO concentrations were assessed in pellet and upper layer at each step of the procedure and MPO was detected in cells by immunocytochemistry. Single-layer colloid centrifugation decreased NSC and MPO concentrations in post-thaw semen. The MPO concentration was correlated with concentration of NSC in the upper layer of the supernatant. In post-thaw semen, with or without previous single-layer colloid centrifugation, MPO concentration was correlated with concentration of NSC. Overall, neutrophils were rarely observed and NSC were mainly epithelial cells or cellular debris, as demonstrated by MPO immunocytochemistry. At all steps of the semen processing and cryopreservation, MPO immunostaining was clearly identified only on NSC. In conclusion, our study shows that NSC present in fresh semen release MPO during freezing.
\end{abstract}

(C) 2013 Elsevier Inc. All rights reserved.

\section{Introduction}

Veterinary and human studies have focused on the impact of oxidation and reactive oxygen species (ROS) on spermatozoa [1,2]. Although the presence of ROS has been shown to be necessary for capacitation and acrosome reaction in bovine sperm [2], an high ROS concentration is known to be

\footnotetext{
* Corresponding author. Tel.: +32 436641 05; fax: +32 43664108 . E-mail address: Jerome.Ponthier@ulg.ac.be (J. Ponthier).
}

deleterious for spermatozoa motility $[3,4]$. Several studies in humans [5-7] and horses [8] have demonstrated an association between ROS and neutrophils in semen. During freezing, when neutrophils are present in the medium, ROS concentrations dramatically increase, even in absence of spermatozoa [7]. Decreased sperm motility and increased ROS concentrations have been observed after incubation of horse semen with neutrophils [8].

Myeloperoxidase (MPO) is an enzyme released by neutrophils during degranulation or lysis $[9,10]$. This peroxidase enzyme catalyzes the reaction of hydrogen peroxide $\left(\mathrm{H}_{2} \mathrm{O}_{2}\right)$ 
and chlorine ions to form hypochlorous acid, an highly oxidant agent [10,11]. Since its purification [12] and the development of an ELISA [9], MPO has been studied in many pathologic pathways $[13,14]$. Myeloperoxidase is present in post-thaw equine semen and its concentration is higher in poor-quality semen $[15,16]$. Implication of non-sperm cells (NSC) in MPO release during freezing has recently been suggested [15,17], but they have not been separated from spermatozoa to assay their MPO content.

Use of single-layer colloid (SLC) centrifugation in silanecoated silica particles solution improved semen quality traits by selecting normal, motile spermatozoa and by decreasing bacterial concentrations $[18,19]$. However, to our knowledge, little is known about effect of centrifugation on NSC concentration.

The objective of this study was to evaluate the effect of SLC centrifugation before cryopreservation on NSC and MPO concentrations in equine semen.

\section{Materials and methods}

\subsection{Animals}

Five healthy, sexually active Quarter Horse stallions with ages ranging from 3 to 19 years were used in the study. The stallions were housed in individual stalls and given daily paddock exercise. They were fed hay and commercial feed to maintain good body condition. Semen was collected every other day over a 2-week period on a phantom using a Missouri-style artificial vagina. The number of mounting attempts to obtain an ejaculation was recorded. For each stallion, four ejaculates were processed and analyzed.

\subsection{Semen evaluation}

Sperm concentration and percentage of sperm with intact plasma membranes were determined using a NucleoCounter SP-100 (Chemometec, Allerod, Denmark) [20] in fresh and post-thaw semen. Total and progressive sperm motility were determined by computerized analysis (CASA; IVOS Version 12.0, Hamilton Thorn Research, Beverly, MA, USA), as previously described [21]. Percent of abnormal sperm forms was obtained by examining 200 spermatozoa after Diff-Quick (RAL, Martillac, France) staining using a light microscopy at an original magnification of $\times 100$. The NSC were also quantified and were categorized as epithelial cells, cellular debris, neutrophils, or others leukocytes (macrophages or lymphocytes) by reading the entire slide's width in three different places. Samples were diluted in formol ( $\mathrm{v} / \mathrm{v}$ dilution) for determining NSC concentration (millions per milliliter) using hemocytometer chambers and phase contrast microscopy at an original magnification of $\times 100$.

\subsection{Semen processing}

A flow chart describing semen processing and sampling is presented in Figure 1. After semen collection, gel was filtered and its volume was subjectively graded from 1 (nearly no gel) to 4 (a large amount of gel). Three aliquots of semen containing $750 \times 10^{6}$ spermatozoa were pipetted into three glass nipple-bottom tubes [21] and topped with semen extender (INRA96; IMV, L'Aigle, France) to obtain a total volume of $25 \mathrm{~mL}$. Thirty microliters of cushion medium (Maxifreeze; IMV*) were gently deposited in the bottom of the nipple tube [21] and the extended semen was

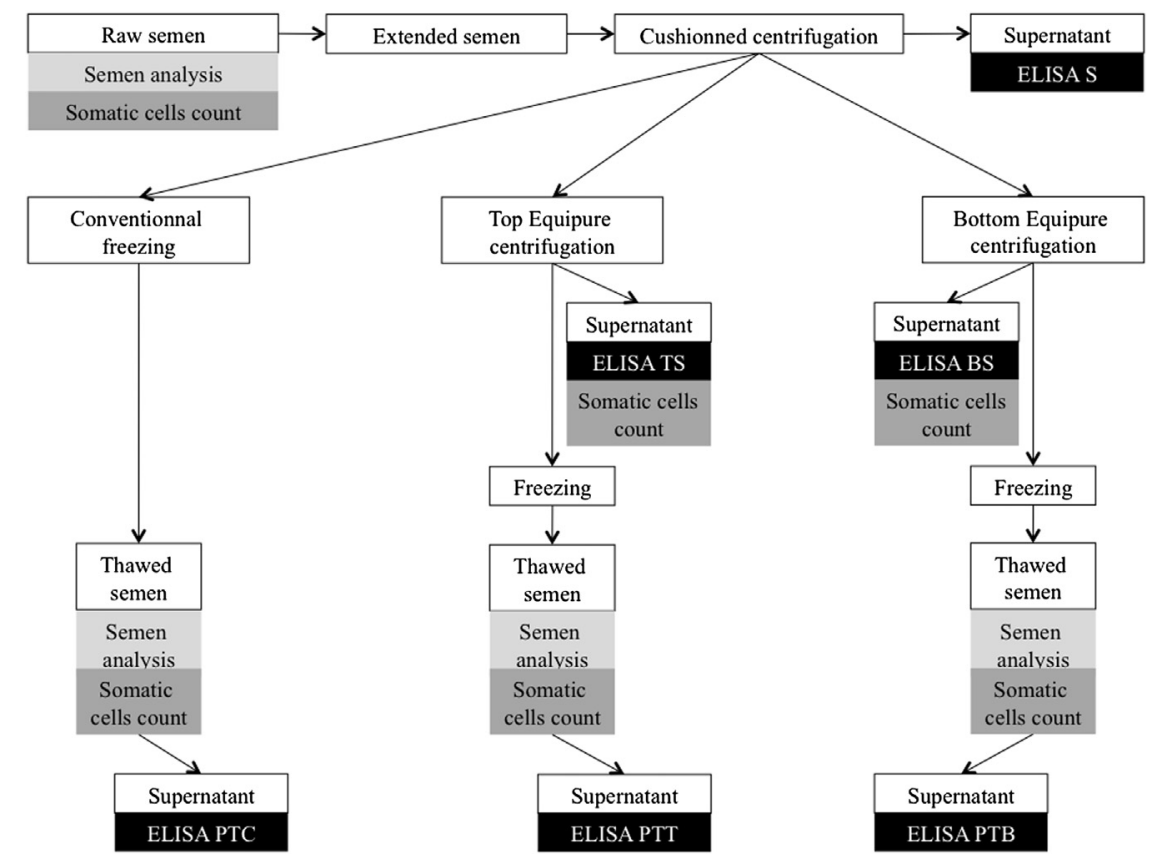

Fig. 1. Flow chart of experimental design. ELISA S, assay on supernatant; ELISA TS, assay on top layer supernatant; ELISA BS, assay on bottom layer supernatant; ELISA PTC, assay on post-thaw conventional semen; ELISA PTT, assay on post-thaw top layer treated semen; ELISA PTB, assay on post-thaw bottom layer treated semen. 
then centrifuged $(400 \times g$ for $20 \mathrm{~min})$. Spermatozoa recovery rate after cushioned centrifugation and NSC recovery rate were calculated respectively as total sperm number post-centrifugation/total sperm number in tube and total NSC number post-centrifugation/total NSC number in tube. After centrifugation, supernatant was removed and stored frozen at $-80^{\circ} \mathrm{C}$ until MPO concentration assay using ELISA (ELISA-F).

One of the three pellets was directly subjected to cryopreservation using the freezing methods described below. The two other pellets were resuspended in $2.2 \mathrm{~mL}$ of INRA 96 extender to obtain a sperm concentration of approximately $300 \times 10^{6}$ spermatozoa $/ \mathrm{mL}$. The concentration of NSC in the resuspended sperm pellets was determined. One milliliter of extended semen was gently pipetted over $3 \mathrm{~mL}$ of either $80 \%$ or $40 \%$ silinated silica-particle colloid solution for density gradient centrifugation (Equipure Bottom Layer or Top Layer, Nidacon International AB, Mölndal, Sweden) in $15-\mathrm{mL}$, conical-bottom tubes and centrifuged $(200 \times \mathrm{g}$ for 30 minutes) as described previously [18].

The supernatant remaining above SLC medium after gradient density centrifugation was then collected. For each sample, NSC concentration was determined using a phase microscope and $2 \mu \mathrm{L}$ were smeared on a slide to assess their presence by immunostaining (described below) and DiffQuick staining. Supernatants collected over SLC medium after centrifugation with top layer (TS) or bottom layer (BS) were then directly frozen at $-80^{\circ} \mathrm{C}$ before being subjected to ELISA assay for MPO concentration.

The three different types of sperm-rich pellets (without treatment, and following top or bottom layer SLC centrifugation) were then processed and frozen using the same methodology. For seminal plasma purification, raw semen was centrifuged $(1800 \times g$ for $10 \mathrm{~min})$ and the resulting supernatant was filtered through 5 - and $1.2-\mu \mathrm{m}$ filters (Millipore, Billerica, MA, USA) to remove residual sperm. Seminal plasma $(5 \%, v / v)$ was then added to the freezing extender (INRA Freeze, IMV). Freezing extender was added to the pellet to obtain a sperm concentration of $100 \times 10^{6}$ spermatozoa $/ \mathrm{mL}$. Straws (0.5-mL, IMV) were then filled and frozen using a programmable freezer (CBS 2100, Custom Biogenic System, Shelby Township, MI, USA) with the following settings: $4{ }^{\circ} \mathrm{C}$ for 75 minutes, then at $-60{ }^{\circ} \mathrm{C} / \mathrm{min}$ to reach $-140^{\circ} \mathrm{C}$. Frozen straws were transferred to liquid nitrogen for storage [22].

Straws were thawed for analysis for 30 seconds in a 37 ${ }^{\circ} \mathrm{C}$ waterbath. After analysis of sperm and NSC concentration, sperm viability, sperm morphology, and sperm motion characteristics, remaining semen was centrifuged $(1500 \times g$ for $10 \mathrm{~min})$ and the supernatant was stored at $-80{ }^{\circ} \mathrm{C}$ for analysis by ELISA. Treatment groups were conventional post-thaw semen (PTC), post-thaw semen processed through top-layer SLC medium (PTT), and postthaw semen processed through bottom SLC medium (PTB).

\subsection{Myeloperoxidase immunocytochemistry assay}

We performed MPO immunostaining as previously described [15] on slides with fresh semen, the two types of gradient centrifugation supernatants (BS and TS), and the three types of thawed semen (PTC, PTT, PTB). Briefly, samples were smeared on a slide and fixed in methanol after drying. After washing in PBS $(3 \times 3 \mathrm{~min})$, the smears were incubated with rabbit anti-MPO antibodies, immunoglobulin $\mathrm{G}$ being diluted at the final concentration of 1.84 $\mu \mathrm{g} / \mathrm{mL}$ with ChemMate Antibody Diluent (Dako Cytomation, Glostrup, Denmark) for 2 hours. Thereafter, the immunostaining was continued with the DakoCytomation LSAB2 System-HRP (DakoCytomation). Control slides were also stained, but without anti-MPO antibodies.

\subsection{Myeloperoxidase ELISA}

We determined MPO concentrations in the extended fresh semen, in BS and TS supernatants, in PTC, PTT, and PTB by commercial ELISA (Bioptis SA, Liège, Belgium) as described previously $[9,16]$. Before analyses, the supernatants were centrifuged again $\left(4^{\circ} \mathrm{C}, 15 \mathrm{~min}, 15,000 \times \mathrm{g}\right)$ to discard the heavy proteins and the cellular fragments. Each sample was assayed twice and the mean value was calculated. For post-thaw semen, concentration was corrected for the dilution by multiplying the concentration by the volume of freezing extender added.

\subsection{Statistical analysis}

Normal distribution of data was tested with Kolmogorov-Smirnov test. Results for normally distributed data were reported as mean values \pm standard deviations. Median values are reported in the case of non-normal distribution. Treatment effects were determined using one-way ANOVA (normally distributed data) or KruskallWallis (non-normally distributed data). Correlations were studied with Pearson or Spearman tests when data were normally or not normally distributed, respectively.

\section{Results}

Ejaculate volume was $47 \pm 22 \mathrm{~mL}$ and sperm concentration was $253 \pm 73 \times 10^{6}$ spermatozoa/mL. Only two ejaculates were obtained after the first mount. Values for initial sperm viability, total motility and progressive motility were $78 \% \pm 9 \%, 77 \% \pm 18 \%$, and $49 \% \pm 15 \%$, respectively. The percentage of morphologically normal spermatozoa in raw semen was influenced by the stallion $(\mathrm{P}<0.01)$. Percent of normal spermatozoa were similar in fresh and PTC semen (75.2\% $\pm 15.1 \%$ and $73.1 \% \pm 17.7 \%$, respectively), but increased $(\mathrm{P}<0.01)$ in PTT and PTB semen $(81.2 \% \pm 13.2 \%$ and $88.5 \% \pm 8.3 \%$, respectively). Overall post-thaw values for sperm concentration, viability, total motility, and progressive motility were $99 \pm 14 \times 10^{6}$ spermatozoa $/ \mathrm{mL}, 51 \% \pm 9 \%$, $42 \% \pm 11 \%$, and $20 \% \pm 9 \%$, respectively. Post-thaw progressive motility was influenced by stallion $(P=.005)$; two stallions had non-freezable semen regardless the use of SLC centrifugation or different freezing extenders.

Mean NSC concentration was influenced by stallion $(\mathrm{P}<$ $0.05)$ and the gel-fraction volume $(P<0.05)$, but not by the number of mounting attempts. No correlation was observed between any of the raw semen quality parameters (concentration, abnormal forms, viability, or progressive and total motility) and NSC concentration in fresh semen. After cushioned centrifugation, the NSC recovery rate was higher 
than that of spermatozoa $(\mathrm{P}<0.01)$. A positive correlation was observed between NSC concentration in fresh and in PTC semen $(r=0.81 ; \mathrm{P}<0.0001)$, but not in thawed semen previously processed with SLC centrifugations. Effect of SLC centrifugations on NSC concentrations in thawed semen is summarized in Figure 2A.

Type of SLC media used for centrifugations did not affect the proportion of NSC types observed in SLC centrifugation supernatants or in thawed semen. In NSC, $78 \%$ (median) were epithelial cells and $21 \%$ were small cellular debris. A small proportion $(<2 \%)$ of neutrophils or other round inflammatory cells were observed. Neutrophils were observed in two ejaculates of two different stallions that were clinically normal. Individual influences were observed in the proportion of epithelial cells $(\mathrm{P}<0.001)$, cellular debris $(\mathrm{P}<$ $0.001)$, and round cells $(\mathrm{P}<0.05)$ in NSC populations.

Immunostaining of gel-free semen showed immunofixation on NSC and no reaction with spermatozoa. Non-sperm cells were not equally stained; large cells were irregularly marked and small cellular debris was highly stained. The background medium was also positively stained with MPOICC, showing a brown halo. The same pattern was observed
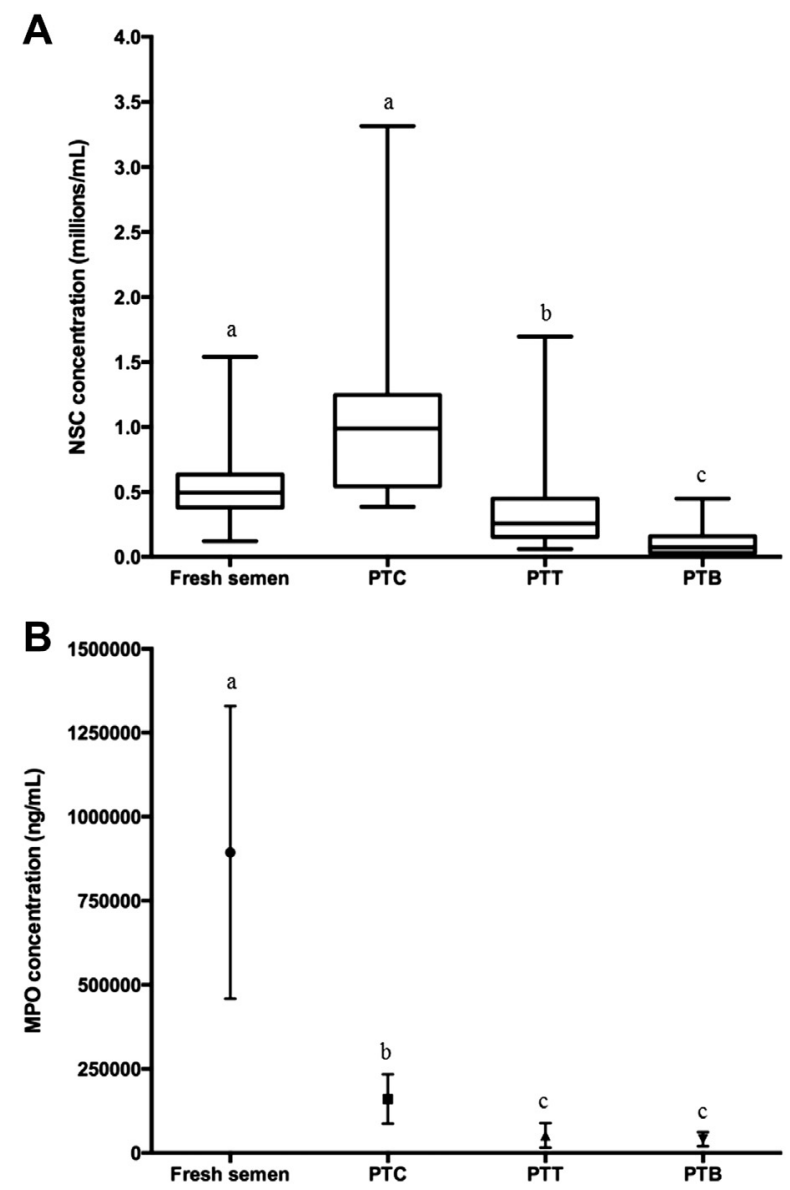

Fig. 2. Median (range) of (A) non-sperm cells (NSC) and mean value ( \pm standard deviation) of (B) myeloperoxidase (MPO) concentrations in fresh and post-thaw equine semen samples. PTC, post-thaw conventional semen; PTT, post-thaw top layer treated semen; PTB, post-thaw bottom layer treated semen. ${ }^{a, b, c}$ Values with different superscripts differ $(P<0.05)$. on slides of SLC centrifugation supernatants; large, nonnucleated cells or those with a pyknotic nucleus were stained, whereas spermatozoa were not. After thawing, immunofixation of large NSC could still be observed, but was more irregular. No immunostaining was observed in negative controls.

There were no individual differences in raw semen MPO concentrations. Semen collection parameters and quality traits (concentration, viability, normal forms ratio, and total and progressive motility) in fresh semen did not correlate with raw semen MPO concentration. The volume of the ejaculate tended to negatively correlate with MPO concentration in fresh semen $(r=-0.55$; $\mathrm{P}=0.054)$. Myeloperoxidase concentrations in fresh and in different types of thawed semen were not correlated. Effect of SLC centrifugations on MPO concentrations in thawed semen is summarized in Figure 2B. Myeloperoxidase median concentration in TB was greater $(P=0.005)$ than in TS. Myeloperoxidase concentration was directly correlated to NSC concentration in SLC centrifugation supernatant $(r=$ 0.47; $\mathrm{P}<0.05)$ and in post-thaw semen $(r=0.39 ; \mathrm{P}<0.05)$.

\section{Discussion}

Semen from two stallions used in this experiment did not freeze satisfactorily with the chosen freezing extender, showing no improvement of progressive motility with PTT and PTB treatments, even if percent of normal spermatozoa increased. Progressive motility for the other three stallions increased after PTT and PTB treatments compared with PTC; percent of normal spermatozoa was increased by PTB. Effect of MPO concentrations on post-thaw motility after SLC should be studied in larger scale studies.

This report confirms that MPO is present in fresh equine semen. Concentrations observed in this experiment were high when compared with other biologic fluids [14,23] and comparable between stallions and with previously published values $[15,16]$. Seminal plasma, which was only partially (5\%) used for cryopreservation, seems to be rich in MPO. Dilution of the MPO with the secretions of the sexual accessory glands could explain the negative correlation observed between MPO concentration and volume of the ejaculate. Vesicular glands are the main contributors to the total volume of the ejaculate [24,25], suggesting that MPO is diluted by vesicular glands secretions.

Neither MPO nor NSC concentrations in fresh semen seem to interfere with quality parameters. In the present study, NSC concentration in fresh semen was influenced by stallion and gel fraction volume. This is consistent with the previously reported presence of NSC in gel fraction [15] and suggests that accessory glands could be involved in NSC release. Non-sperm cell concentration in untreated thawed semen (PTC) was directly correlated with that of fresh semen and NSC recovery rate was higher than spermatozoa recovery rate after centrifugation. This finding is easily explained by the immobility of the NSC, but also suggests that conventional freezing processing increases NSC concentration. As expected, SLC centrifugation significantly decreased somatic cells concentration and lowest NSC concentration was achieved after bottom-layer SLC centrifugation (PTB). These data agree with previous publications 
assessing the removal of cellular debris in horses [18] and of bacteria in human [26].

Type of SLC gradient medium (bottom vs. top) had no effect on proportions of cellular types observed. Cytologic observations in all types of samples (SLC centrifugation supernatants, fresh and thawed semen) revealed predominantly epithelial cells or cellular debris, with various degrees of keratinization and nuclear pyknosis, as described previously [15]. A stallion effect was observed on epithelial cells and cellular debris proportions, which might illustrate a difference in NSC release or degeneration between stallions. As described previously [15], large epithelial NSC were irregularly stained MPO immunofixation and small cellular debris where highly marked, whereas no MPO immunostaining was observed on spermatozoa. To date, few data are available about the origin and characterization of NSC in the equine. In dogs, squamous epithelial cells are observed in prostatic squamous metaplasia [27,28] and, recently, MPO has been observed in epithelial cells of human prostatic biopsies [29]. Further investigation in the equine should consider specific staining of genital tract biopsy samples to assess NSC anatomic origin.

Concentrations of MPO and NSC were correlated in SLC centrifugation supernatants and in post-thaw semen, as suggested recently [17]. Associations between MPO and NSC concentrations indicate an MPO release by these cells in semen when temperature decreases [15]. This observation explains the increase of MPO observed in all post-thaw samples, containing only $5 \%$ of seminal plasma. As described in laminitis [30-32], MPO immunostained epithelial cells could have secondarily absorbed MPO released during a genital tract inflammation, although neutrophils could no longer be observed because they have rapidly undergone apoptosis [9]. It can be speculated that NSC undergoing cellular damage associated with freezing and thawing procedures [7,33] could release MPO, somehow acting like sponges. Further research should focus on gaining a better understanding of the timing and localization of the incorporation of MPO in NSC.

In conclusion, our study shows that NSC are present in fresh semen and release MPO during freezing, which has been shown to be negatively associated with post-thaw semen quality. These results show that NSC observed in equine semen could be deleterious during freezingthawing processes. Larger studies combining effect of MPO and NSC on post-thaw motility should be conducted.

\section{Acknowledgments}

The authors thank Prof. Johann Detilleux for the statistical assistance, Ms. Ariane Niesten (CORD) for the ELISA assays and MPO-ICC staining, and Ms. Katrina LaCaze, Dr Justin Voge, and Dr. Shelby Hayden (Texas A\&M University) for assistance during collection and freezing procedures.

\section{References}

[1] Agarwal A, Ikemoto I, Loughlin KR. Relationship of sperm parameters with levels of reactive oxygen species in semen specimens. J Urol 1994;152:107-10.
[2] O’Flaherty CM, Beorlegui NB, Beconi MT. Reactive oxygen species requirements for bovine sperm capacitation and acrosome reaction. Theriogenology 1999;52:289-301.

[3] Baumber J, Ball BA, Gravance CG, Medina V, Davies-Morel MC. The effect of reactive oxygen species on equine sperm motility, viability, acrosomal integrity, mitochondrial membrane potential, and membrane lipid peroxidation. J Androl 2000;21:895-902.

[4] Peris SI, Bilodeau JF, Dufour M, Bailey JL. Impact of cryopreservation and reactive oxygen species on DNA integrity, lipid peroxidation, and functional parameters in ram sperm. Mol Reprod Dev 2007;74: 878-92.

[5] Henkel R, Kierspel E, Stalf T, Mehnert C, Menkveld R, Tinneberg HR, et al. Effect of reactive oxygen species produced by spermatozoa and leukocytes on sperm functions in non-leukocytospermic patients. Fertil Steril 2005;83:635-42.

[6] Wang A, Fanning L, Anderson DJ, Loughlin KR. Generation of reactive oxygen species by leukocytes and sperm following exposure to urogenital tract infection. Arch Androl 1997;39:11-7.

[7] Wang AW, Zhang H, Ikemoto I, Anderson DJ, Loughlin KR. Reactive oxygen species generation by seminal cells during cryopreservation. Urology 1997;49:921-5.

[8] Baumber J, Vo A, Sabeur K, Ball BA. Generation of reactive oxygen species by equine neutrophils and their effect on motility of equine spermatozoa. Theriogenology 2002;57:1025-33.

[9] Franck T, Grulke S, Deby-Dupont G, Deby C, Duvivier H, Peters F, et al. Development of an enzyme-linked immunosorbent assay for specific equine neutrophil myeloperoxidase measurement in blood. J Vet Diagn Invest 2005; 17:412-9.

[10] Serteyn D, Grulke S, Franck T, Mouithys-Mickalad A, DebyDupont G. La myeloperoxydase des neutrophiles, une enzyme de défense au capacité oxydantes [Neutrophil myeloperoxidase, an oxidant defensive enzyme]. Annales de Médecine Vétérinaire 2003; 147:79-93.

[11] Taurog A, Dorris ML. Myeloperoxidase-catalyzed iodination and coupling. Arch Biochem Biophys 1992;296:239-46.

[12] Mathy-Hartert M, Bourgeois E, Grulke S, Deby-Dupont G, Caudron I, Deby $C$, et al. Purification of myeloperoxidase from equine polymorphonuclear leucocytes. Can J Vet Res 1998;62:127-32.

[13] de la Rebiere G, Franck T, Deby-Dupont G, Salciccia A, Grulke S, Peters F, et al. Effects of unfractionated and fractionated heparins on myeloperoxidase activity and interactions with endothelial cells: possible effects on the pathophysiology of equine laminitis. Vet J 2008; 178:62-9.

[14] Grulke S, Franck T, Gangl M, Peters F, Salciccia A, Deby-Dupont G, et al. Myeloperoxidase assay in plasma and peritoneal fluid of horses with gastrointestinal disease. Can J Vet Res 2008;72:37-42.

[15] Ponthier J, Desvals M, Franck T, de la Rebiere de Pouyade G, Spalart M, Palmer E, et al. Myeloperoxidase in equine semen: concentration and localization during freezing processing. J Equine Vet Sci 2012;32:32-7.

[16] Ponthier J, Franck T, Detilleux J, Mottart E, Serteyn D, Deleuze S. Association between myeloperoxidase concentration in equine frozen semen and post-thawing parameters. Reprod Domest Anim 2010;45:811-6.

[17] Ponthier J, de la Rebiere de Pouyade G, Desvals M, Spalart M, Franck T, Serteyn D, et al. Is neutrophil elastase associated with myeloperoxidase concentration and post-thawing parameters in equine frozen semen? Anim Reprod Sci 2010;121S:S200-2.

[18] Edmond AJ, Teague SR, Brinsko SP, Comerford KL, Waite JA, Mancill SS, et al. Effect of density-gradient centrifugation on quality and recovery rate of equine spermatozoa. Anim Reprod Sci 2008; 107. 318.

[19] Villegas J, Schulz M, Soto L, Iglesias T, Miska W, Sanchez R. Influence of reactive oxygen species produced by activated leukocytes at the level of apoptosis in mature human spermatozoa. Fertil Steril 2005; 83:808-10.

[20] Johansson CS, Matsson FC, Lehn-Jensen H, Nielsen JM, Petersen MM. Equine spermatozoa viability comparing the NucleoCounter SP-100 and the eosin-nigrosin stain. Anim Reprod Sci 2008;107:325-6.

[21] Waite JA, Love CC, Brinsko SP, Teague SR, Salazar Jr JL, Mancill SS, et al. Factors impacting equine sperm recovery rate and quality following cushioned centrifugation. Theriogenology 2008;70:704-14.

[22] Pillet E, Duchamp G, Batellier F, Beaumal V, Anton M, Desherces S, et al. Egg yolk plasma can replace egg yolk in stallion freezing extenders. Theriogenology 2011;75:105-14.

[23] Art T, Franck T, Lekeux P, de Moffarts B, Couetil L, Becker M, et al. Myeloperoxidase concentration in bronchoalveolar lavage fluid from healthy horses and those with recurrent airway obstruction. Can J Vet Res 2006;70:291-6. 
[24] Amann RP. Functional anatomy of the adult male. In: McKinnon AO, Squires EL, Vaala WE, Varner DD, editors. Equine reproduction. Philadelphia: Lea \& Febiger; 1993. p. 645-57.

[25] Brinsko SP. Retrograde ejaculation in a stallion. J Am Vet Med Assoc 2001;218:551-3.

[26] Villegas J, Schulz M, Soto L, Sanchez R. Bacteria induce expression of apoptosis in human spermatozoa. Apoptosis 2005;10:105-10.

[27] Johnston SD, Kamolpatana K, Root-Kustritz MV, Johnston GR. Prostatic disorders in the dog. Anim Reprod Sci 2000;60-61: 405-15.

[28] Smith J. Canine prostatic disease: a review of anatomy, pathology, diagnosis, and treatment. Theriogenology 2008;70:375-83.

[29] Roumeguere T, Delree P, Van Antwerpen P, Rorive S, Vanhamme L, de Ryhove LD, et al. Intriguing location of myeloperoxidase in the prostate: a preliminary immunohistochemical study. Prostate 2012; 72:507-13.

[30] Black SJ. Extracellular matrix, leukocyte migration and laminitis. Vet Immunol Immunopathol 2009;129:161-3.

[31] de la Rebiere de Pouyade G, Riggs LM, Moore JN, Franck T, DebyDupont G, Hurley DJ, et al. Equine neutrophil elastase in plasma, laminar tissue, and skin of horses administered black walnut heartwood extract. Vet Immunol Immunopathol 2010;135:181-7.

[32] Riggs LM, Franck T, Moore JN, Krunkosky TM, Hurley DJ, Peroni JF, et al. Neutrophil myeloperoxidase measurements in plasma, laminar tissue, and skin of horses given black walnut extract. Am J Vet Res 2007;68:81-6.

[33] Graham-Pole J, Davie M, Willoughby ML. Cryopreservation of human granulocytes in liquid nitrogen. J Clin Pathol 1977;30:758-62. 